\title{
Comparison of approximate intermolecular potentials for ab initio fragment calculations on medium sized N-heterocycles
}

\author{
Bónis Barcza, Ádám B. Szirmai, Katalin J. Szántó, Attila Tajti, Péter G. Szalay*
}

December 17, 2021

\begin{abstract}
The ground state intermolecular potential of bimolecular complexes of N-heterocycles is analysed for the impact of different terms of the interaction energy as provided by various, conceptually different theories. Novel combinations with several formulations of the electrostatic, Pauli repulsion, dispersion and other contributions are tested for a good performance at both short- and long-distance sides of the potential energy surface for various alignments of the pyrrole dimer as well as the cytosine-uracil complex. The integration of a DFT/CC density embedding scheme and dispersion terms from the effective fragment potential (EFP) method is found to provide very good agreement with the reference $\operatorname{CCSD}(\mathrm{T})$ potential overall, but a $\mathrm{QM} / \mathrm{MM}$ approach using CHELPG atomic point charges for the electrostatic interaction augmented by EFP dispersion and Pauli repulsion contributions comes also close. Both of these schemes has the advantage of not relying on predefined force fields, rather the interaction parameters can be obtained for the system under study, therefore excellent candidates for $a b$ initiomodeling.
\end{abstract}

Keywords: intermolecular interactions, Effective Fragment Potential, Pauli repulsion, embedding, QM/MM, dispersion

*ELTE Eötvös Loránd University, Institute of Chemistry, Laboratory of Theoretical Chemistry, P. O. Box 32, H-1518, Budapest 112, Hungary, e-mail: szalay@chem.elte.hu 


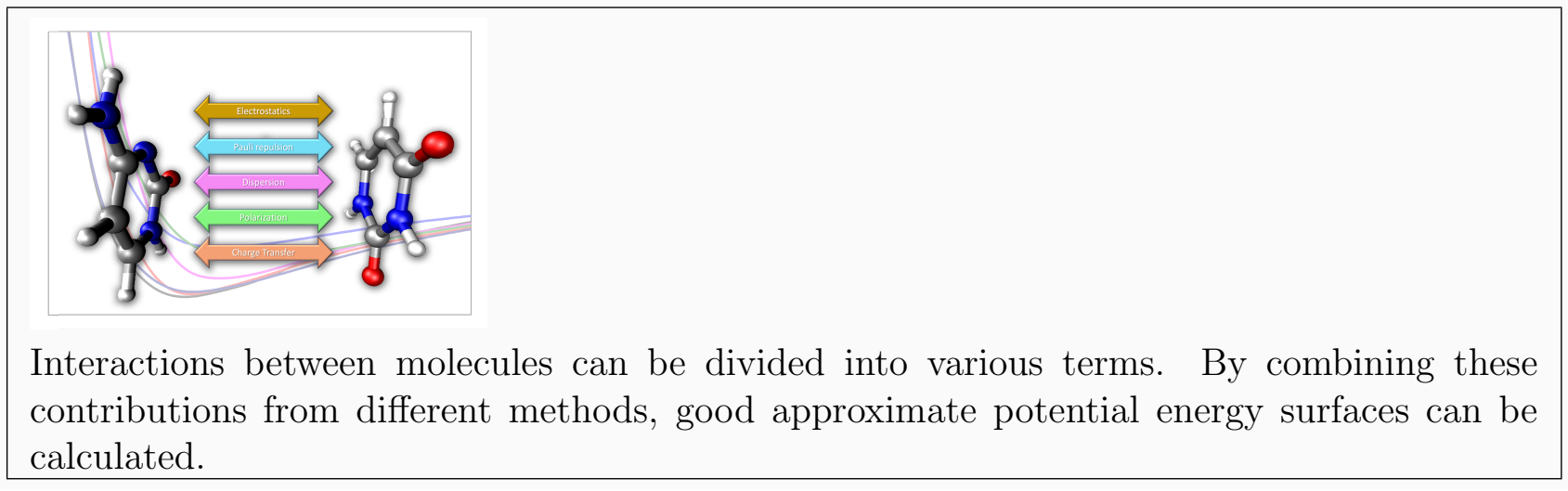




\section{Introduction}

Ab initio quantum chemistry calculations are now indispensable part of chemical research. Although there is a large arsenal of methods available for such investigations, the cost of the calculations grows with the system size rapidly: the more advanced the method is the faster the cost of the calculations grows. A possible way to overcome this problem is the fragmentation of the system into smaller components that can be treated reliably by computational methods and a formulation to account for their interaction. The number of methods of this kind is tremendous, yet there are still many open challenges associated with them. One of the biggest such issues is related to the need to cut through chemical bonds during the fragmentation procedure.

A much simpler situation for fragmentation methods is presented by systems where the fragments are real molecules connected with non-covalent interactions. In this case the Hamiltonian of a two-component system is trivially partitioned as

$$
\hat{H}\left(r_{1}, r_{2}\right)=\hat{H}_{1}\left(r_{1}\right)+\hat{H}_{2}\left(r_{2}\right)+\hat{V}\left(r_{1}, r_{2}\right)
$$

with $\hat{H}_{i}$ being the Hamiltonian of the non-interacting fragments, while the $\hat{V}\left(r_{1}, r_{2}\right)$ operator is responsible for describing the interaction. This form readily suggests perturbation theory as the approach of choice to describe the interaction, with the zeroth order wave function being the product of the fragments' wave functions.

A large variety of methods with this starting ansatz are available for routine calculations today, with a wide spectrum of theoretical sophistication. Many of these theories offer the division of the interaction energy into various, physically interpretable terms, including the purely electrostatic, dispersion, Pauli repulsion and other forces. The relative importance of these contributions is highly dependent on the systems under investigation as well as their distance. The possible methods used for non-covalent interaction can be classified as follows:

- Symmetry-Adapted Perturbation Theory (SAPT)theory, ? which is in fact a hierarchical set of methods based on double perturbation theory with respect to interaction terms and electron correlation. SAPT allows the calculation of the magnitude of different, physically motivated contributions, as well as the effect of electron correlation on them. These methods are often used as reference in benchmarking studies. 
- Classical force fields readily offer pre--calculated, transferable parameter sets for the evaluation of the most important contributions to the non-covalent interaction energy such as electrostatic terms (partial charges, atomic multipoles), as well as LennardJones type terms (accounting for dispersion and Pauli repulsion).

- Methods calculating the interaction energy directly from properties of the monomers. Among the many methods of this type, the EFP (Effective Fragment Potential) methods $?$ are the most prominent.

- Embedding type methods where the interaction is included in the Hamiltonian of the fragment(s). The most widespread in this respect are the so called $\mathrm{QM} / \mathrm{MM}$ approaches, ${ }^{?}$ but other density-based embedding methods can also be used.

The authors of this paper have the long term focus on the reliable description of the interaction of fragment-localized chromophores, in which, as preliminary calculations showed, the most often used purely electrostatic approximations fail to provide the correct potential energy surface of the interacting excited states due to the importance of other contributions that are missing from this picture. ${ }^{?}$ This is particularly true when the short-distance part of the potential energy surfaces is of interest, e.g. for stacked interactions in DNA.

To find the best suited approximation for these terms, in this paper we investigate the ground state of non-covalently interacting bimolecular complexes with the aim of comparing various approximative methods for different contributions of the interaction energy. Keeping our focus on nitrogen-containing heterocycles, as test systems we use pyrrole dimers (denoted hereafter as Pyr-Pyr) in different orientations as well as the cytosine-uracil base pair (Cyt-Ura) in stacked form. These complexes have low lying Frenkel-type pairs of excited states where the proper handling of the non-covalent interaction is very important to obtain accurate splittings.

The paper is organized as follows. In Section ?? we give a short summary of the available methodologies describing non-covalent interactions, concentrating on the differences in theoretical formulations and the possibility of incorporation into $a b$ initiocalculations. On the basis of this analysis in Section ?? we select a set of methods used in the tests. Section ?? describes the computational details, while Section ?? presents the results. 


\section{Methodology of modeling intermolecular interactions}

Already at the dawn of quantum chemical treatment of molecules, it became clear that quantum mechanics gives rise to important short-range forces ${ }^{\text {? }}$ and the treatment of intermolecular interactions has now a broad background in theory. Systematic overviews of this topic were presented in comprehensive texts. ? ? ? ?

The contributions to the interaction energy are usually called ? ?

- Electrostatic (eventually including polarization effects);

- Dispersion;

- Pauli repulsion (exchange repulsion);

- Charge Transfer (CT);

- and combinations thereof.

One way to distinguish these terms is offered by the SAPT theory ${ }^{?}$ which in different orders of the perturbation theory provides approximate definitions for the respective terms of the interaction energy. One should always keep in mind, however, that distinguishing these contributions is non-physical since an exact (or accurate) calculation on the complete system would give just one single, interaction energy'. Nevertheless, from the perspective of chemical and physical intuition, and also for developing reasonable approximations, this distinction is highly warranted and helpful.

In SAPT the electrostatic contribution of the interaction energy comes as first order in the interaction operator, with sizable contributions from the correlation terms. ? Thus its careful modelling is essential in order to get accurate results.

The electrostatic interaction can traditionally be incorporated into the model via charges placed on the interacting molecules. ? If these charges are at the atomic positions, the model has a strong link to chemical intuition since, in the simplest case, the charges represent the partial charges on the atoms. Also this choice allows for transferable force fields since it is possible to assign charges to certain atom types, a concept used for other (intramolecular) pa- 
rameters of the force fields, as well. Traditional force fields like AMBER ? ? or CHARMM? use such parametrization.

The use of partial charges in QM/MM type approaches is quite popular, due to the easy incorporation of their effect into the one-electron operator of most QM codes. ${ }^{\text {? }}$ The ways of defining the partial charges have been reviewed by Janeček et al. ${ }^{\text {r }}$ recently. Here we just mention that the population analysis concept normally used in earlier times is nowadays replaced by fitting directly to interaction energies, dipole moments or electrostatic potential

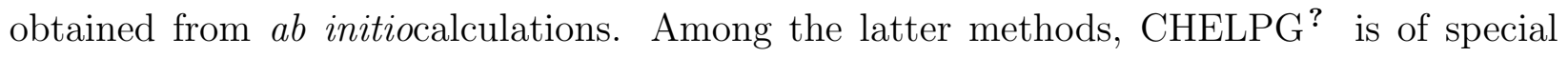
importance for the present study since it represents a readily available algorithm to calculate the charges for the particular monomer of interest. A recent advance in this field is the $\mathrm{W}$ RESP potential of Janeček et al. ?

A more flexible and therefore more accurate parametrization of the electrostatic interaction can be achieved using atomic multipoles, first suggested by Stone. ? Pioneering work in applying this technique was done in the Effective Fragment Potential (EFP) frame-

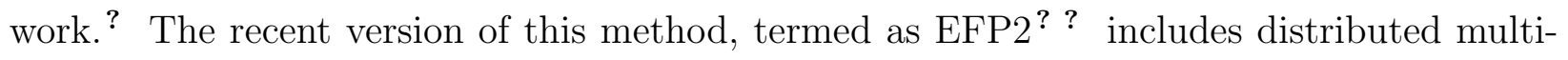
poles up to octupoles at the atomic centers and also at bond midpoints, while polarizability is included for localized molecular orbitals. ${ }^{?}$ Modern force fields like AMOEBA (Atomic Multipole Optimized Energetics for Biomolecular Applications), ${ }^{?}$ SIBFA (Sum of Interactions Between Fragments Ab initio computed), ? ? DRF (Discrete Reaction Field) ${ }^{\text {also }}$ use this parametrization including multipoles up to quadrupoles, often also with polarizable components.

For very short intermolecular distances however, the direct application of the classical electrostatic interactions, either in terms of charges or multipoles, does not work: the overlap of the electronic densities cannot be described correctly by discrete charge distributions. The importance of this effect for example, in the case of the stacked nucleobases was shown by Sherill and co-workers? ? in a comparison to SAPT0: the slightly repulsive electrostatic interaction turns into an attraction below $4 \AA$ at the SAPT0 level, while AMBER and CHARMM remain repulsive. This effect has an important role of compensating e.g. Pauli repulsion (see later). To cover this artifact, damping terms have been introduced? in several model potentials like EFP, ${ }^{?}$ AMOEBA, ? ? ${ }^{\text {SIBFA, }},{ }^{?}$ or as a stand-alone extension, ${ }^{?}$ 
as well as implemented in the Gaussian program package? for QM/MM type electrostatic modeling.

Additional two energy components decay fast with the distance of the fragments but proved to be important also in SAPT theory: ? The dispersion energy which is due to the correlation of electrons in the two fragments, respectively, comes first in second order of perturbation theory and correlation contributions are clearly essential. Pauli exchange repulsion energy is obtained when antisymmetrizing the wave function of the entire system.

In molecular force fields dispersion and Pauli repulsion are often considered together as a van der Waals potential, which could be traced back to the suggestion of LennardJones. ' A comprehensive historical summary of the treatment of these terms was published

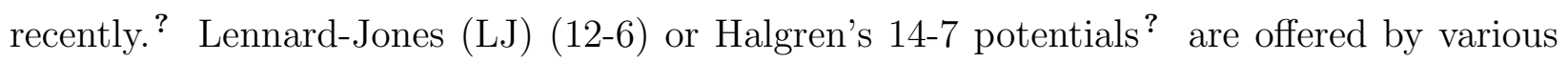
force fields for the inclusion of the van der Waals interaction, i. e. for dispersion and Pauli exchange combined. More sophisticated models are also available which use a more rigorous theoretical formulation based on monomer properties. For example, $\mathrm{S}^{2}$-dependent functions (with S being the orbital overlap) for the Pauli repulsion is used in SIBFA ? and EFP. ? ?

For dispersion, EFP2 calculates the so-called $\mathrm{C}_{6}$ coefficient of the London expansion as the instantaneous dipole-induced-dipole interaction, obtained from the frequency dependent polarizabilities and also includes a $\mathrm{C}_{8}$ term estimated as one-third of the $\mathrm{C}_{6}$ one. ${ }^{\text {? The }}$ different versions of the D correction (D, D2, D3) by Grimme et al. ? ? ? were developed to correct for the missing dispersion correction in DFT methods, but can readily be used for intermolecular dispersion as well. Similarly to the respective EFP2 term, it is based on a London expansion up to $\mathrm{C}_{8}$ with the $\mathrm{C}_{6}$ calculated from hydrides of the given atoms. It also includes a theoretically justifiable damping factor and three-body terms.

Over these a posteriori calculations of the different terms, a more sophisticated way for the consideration of intermolecular interactions is the inclusion of the interaction potential in the quantum mechanical Hamiltonian of the fragment. These methods belong to the general family of embedding methods which offer a large variety of solutions to the problem at hand. ' In these schemes the subsystem(s) of interest is (are) described by higher levels of theory, while their environment is approximated in a simpler manner. Such methods include embedding into environment described as continuum ${ }^{\text {? }}$, molecular mechanics (MM) ${ }^{?}$ or 
quantum mechanics (QM) ${ }^{\text {? }}$

The simplest embedding scheme, resulting in methods often referred to as QM/MM, is when the quantum system is embedded into a molecular mechanics environment. In this case the QM Hamiltonian of a fragment is augmented by (possibly one-electron) interaction terms usually obtained from force fields. The point charges offered by the force fields can readily be used in this scheme to include the electrostatic contribution, ? ? but the inclusion of multipoles is also possible, see for example the QM/EFP2 method. ' An advantage of such $\mathrm{QM} / \mathrm{MM}$ approaches is that in principle one can use any QM level to calculate the fragment's electronic structure under the influence of the environment? and even the treatment of excited states is possible in a simplified manner.

The inclusion of Pauli repulsion and dispersion is less straightforward in QM/MM calculations. Therefore, very often these are just used as a posteriori corrections to the energy. One should realize, however, that such potentials depend only on the distance (of the atoms), the parameters are pre-defined and thus do not reflect the exact electronic structure of the fragments. In particular, the potentials are obtained for ground states, thus their use for excited states is not entirely justified.

To overcome these problems, recently an embedding version of EFP2 method, termed as QM/EFP2 or ab initio/EFP2 has been introduced. ? ? By this development, inclusion of the dispersion? and the Pauli exchange? terms is possible in the form of special oneelectron operators. Ref. ? compares individual terms obtained with EFP2 and QM/EFP2 procedures with the corresponding SAPT contributions for the S66 test set. ${ }^{\text {It }}$ ' was found that QM/EFP2 does not necessarily deliver superior interaction potentials, which is most probably due to the lesser error compensation compared to the regular EFP model. First applications to excited states give promising results. ? Implementations of QM/EFP are available in the GAMESS? and Q-Chem? program suites.

The embedding scheme also offers various ways for treating all subsystems at the QM level. The main advantage of such methods is that the interaction with the electron density of the environment can directly be calculated without discretization of the charge distribution or parametrization of other contributions. Usually, the density of the surroundings is calculated at a lower level of theory, resulting in substantial computational savings. The 
Kohn-Sham (KS) density functional theory (DFT) offers an excellent framework for this QM based embedding as DFT scales favourably with the system size while providing a relatively accurate description of the environment's density. In a typical DFT embedding scheme the energy of the system can be written as

$$
E[\rho]=E\left[\rho_{\mathrm{A}}\right]+E\left[\rho_{\mathrm{B}}\right]+E_{\mathrm{int}}\left[\rho_{\mathrm{A}}, \rho_{\mathrm{B}}\right]
$$

where A is the ,,active" subsystem of interest, B is the approximated environment, the third term contains the interaction between $\mathrm{A}$ and $\mathrm{B}$, while $\rho$ is the density of the total system or that of the subsystem denoted in the subscript. The main issue with DFT embedding is the treatment of kinetic energy contributions appearing in the interaction term that are not additive over the subsystems. This non-additive kinetic energy can be eliminated by enforcing orthogonality between the orbitals of the subsystems. Several methods, including projector based approaches like the level shift method of Manby et al. ${ }^{?}$ and the Huzinaga

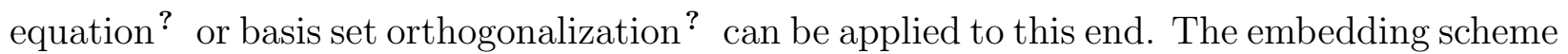
based on the Huzinaga approach by Hégely and co-workers ? ? is especially appealing since it provides a simple and formally exact framework and the active site(s) can also be described by wave function (WF) methods, allowing eventually the treatment of excited states as well. This technique employs a subtractive embedding scheme where a KS-DFT calculation is performed on the entire system which is then divided into an active and an environment part. The energy of an embedding calculation of this type can be given as

$$
E_{\text {tot }}^{\mathrm{WF}-\mathrm{in}-\mathrm{DFT}}=E_{\mathrm{tot}}^{\mathrm{DFT}}-E_{\mathrm{A}}^{\mathrm{DFT}}+E_{\tilde{\mathrm{A}}}^{\mathrm{WF}}
$$

where $E_{\tilde{\mathrm{A}}}^{\mathrm{WF}}$ is calculated in the presence of the environment's density, including thereby the electrostatic interaction of the subsystems. Note that the exchange interaction between the two subsystems is retained from the original supersystem calculation and included in the final energy. However, since the underlying DFT calculation on the supersystem does not describe dispersion correctly, this contribution needs to be added afterwards.

In this work we test various methods belonging to the main categories: a posteriori corrections sourced from some representative force fields including also EFP2, which can formally be considered as a system-specific FF, as well as embedding techniques. Embedding methods beyond QM/MM were, to our knowledge, not yet studied systematically for 
intermolecular interactions. From the numerical study presented in this paper we exclude SAPT, which is certainly not justified in case of the ground state. However, SAPT is not a viable choice for excited states where the results of this study are intended to be transferred to: as of today, to our knowledge, SAPT is not set to be generally used for excited state problems, although one should acknowledge here a recent promising effort by Hapka et al.?

\section{Interaction potentials}

To fulfill the research goals outlined in the introduction, in this work we test various methods summarized in the Methodology section in order to obtain information about how these describe the different contributions of the non-covalent interaction energy. The aim is to find a combination of these contributions which is accurate enough for the ground state of the molecular systems we are interested in, can be used in combination with various QM methods, and eventually can also be applied for excited states. Our study is pragmatic, in the sense that instead of seeking general conclusions for the different methods, we intend to identify the best potentials/procedures to describe complexes of N-heterocycles at the vicinity of their equilibrium structures.

Table ?? summarizes the methods we explore to describe various contributions to the intermolecular interaction energy. 
Table 1: Overview of the methods and combinations used in this study.

\begin{tabular}{|c|c|c|c|c|c|}
\hline \multirow{2}{*}{ Method } & \multicolumn{5}{|c|}{ Contribution } \\
\hline & Electrostatics & Pauli repulsion & Dispersion & Polarization & $\mathrm{CT}$ \\
\hline GAFF Point Charges $(G A F F Q M / M M)$ & $\bullet$ & & & & \\
\hline CHELPG Point Charges (CHELPG $Q M / M M)$ & $\bullet$ & & & & \\
\hline EFP2 Multipole Expansion (EFP2 $E l$ ) & $\bullet$ & & & & \\
\hline Huzinaga embedding (Embed) & $\bullet$ & $\bullet$ & & & \\
\hline EFP2 Pauli repulsion (EFP2 Rep) & & $\bullet$ & & & \\
\hline EFP2 Dispersion (EFP2 Disp) & & & $\bullet$ & & \\
\hline D3 Correction (D3 Disp) & & & $\bullet$ & & \\
\hline GAFF Lennard-Jones $(G A F F L J)$ & & $\bullet$ & $\bullet$ & & \\
\hline EFP2 Polarization (EFP2 Pol) & & & & $\bullet$ & \\
\hline EFP2 Charge Transfer $(E F P 2 C T)$ & & & & & $\bullet$ \\
\hline Total EFP2 (EFP2 Tot) & $\bullet$ & $\bullet$ & $\bullet$ & $\bullet$ & $\bullet$ \\
\hline \multicolumn{6}{|c|}{ Combinations } \\
\hline$\left(E F P^{2} E l+E F P 2 R e p\right)$ & $\bullet$ & $\bullet$ & & & \\
\hline$(E F P \mathscr{2} R e p+E F P \mathscr{2}$ Disp $)$ & & $\bullet$ & $\bullet$ & & \\
\hline$\left(E F P^{2} E l+E F P 2 R e p+E F P 2\right.$ Pol $\left.+E F P^{2} C T\right)$ & $\bullet$ & $\bullet$ & & $\bullet$ & $\bullet$ \\
\hline$\left(E F P^{2} E l+E F P 2 \operatorname{Rep}+E F P^{2}\right.$ Disp $)$ & $\bullet$ & $\bullet$ & $\bullet$ & & \\
\hline$(C H E L P G Q M / M M+G A F F L J)$ & $\bullet$ & $\bullet$ & $\bullet$ & & \\
\hline$($ CHELPG QM/MM + EFP2 Rep + EFP2 Disp) & $\bullet$ & $\bullet$ & $\bullet$ & & \\
\hline$($ Embed + D3 Disp $)$ & $\bullet$ & $\bullet$ & $\bullet$ & & \\
\hline$($ Embed + EFP2 Disp $)$ & $\bullet$ & $\bullet$ & $\bullet$ & & \\
\hline
\end{tabular}

\section{Computational details}

\subsection{Ab initio calculations}

The reference energy was obtained using the $\operatorname{CCSD}(\mathrm{T})$ method with the aug-cc-pVDZ basis ${ }^{\text {? }}$ and applying counterpoise (CP) correction? in order to overcome the basis set superposition error. The choice of this relatively small basis set is justified by previous findings of Sinnokrot et al. ${ }^{?}$ whose results confirmed that, if used with the CP correction, the aug-cc-pVDZ basis provides good agreement with larger basis sets in case of stacked interactions.

The core electrons were excluded from the correlation treatment.

The QM/MM calculations were performed at the CCSD/aug-cc-pVDZ level using the CFOUR ${ }^{?}$ ? program package, with the MM point charges incorporated into the one-electron 
Hamiltonian. The FF electrostatic point charges, as well as the parameters of the tested Lennard-Jones potential were taken from the General Amber Force Field (GAFF) ${ }^{\text {? }}$. The values of the FF parameters for the investigated systems are shown in the Supplementary material.

The calculation of CHELPG point charges for the QM/MM calculations were performed using the GAMESS? program system at the CCSD/aug-cc-pVDZ level. The electrostatic potential of the molecule was calculated on a set of grid points around the molecule, with the distance between these points being $0.8 \AA$ and the most distant point from any atom set to $3 \AA$. The magnitude of point charges placed on the atoms was fitted to reproduce this potential, with the additional constraint to reproduce also the dipole and quadrupole moments of the molecule. The values of the CHELPG atomic charges obtained this way can also be found in the Supplementary material.

The EFP2 interaction potentials for the molecules were calculated using the GAMESS built-in routine with the default settings. The parameters used include the fitted multipole moments up to octupoles along with exponential damping functions to account for the charge penetration effects, as well as dipole polarizabilities and unique sets of parameters for Pauli repulsion, dispersion and charge transfer interactions. All of these were obtained from a restricted Hartree-Fock calculation on the individual molecules using the $6-311++\mathrm{G}(3 \mathrm{df}, 2 \mathrm{p})$ basis set, following the previous choice of Slipchenko and Gordon? for different orientations of the benzene dimer.

For the DFT/WF embedding calculations we used the Huzinaga embedding scheme of Hégely and co-workers. ? In this approach first a KS-DFT calculation is performed on the entire system which is then divided into an active and an environment part. The orbitals are then localized on the subsystems and those on the active subsystem are reoptimized by solving the Huzinaga equation which includes the interaction with the environment represented by its occupied orbitals. These optimized orbitals were subsequently used in the wave function (WF) calculation on the active subsystem. The localization and partitioning of the orbital spaces plays an important role in this scheme: not only does it serve the purpose of separating the occupied orbitals of the active subsystem and the environment, but the localization of the virtual orbitals truncates the supersystem's orbital space to the orbital 
space of the active subsystem, making the computational cost of the ab initio correlated calculations more favourable. This localization is done using the Subsystem Projected AO Decomposition (SPADE) ${ }^{?}$ approach, which works in a black-box fashion, only requiring the atoms to be assigned to the respective subsystems.

These embedding calculations were also performed with the aug-cc-pVDZ basis set, using the MRCC program system. ? ? For the KS-DFT part of the calculations the PBE ${ }^{\text {? }}$ functional was employed, while the subsequent WF calculation on the active subsystem was done at the CCSD level with the core electrons uncorrelated.

The PBE-D3 dispersion correction? was evaluated using the DFTD3 program with Becke-Johnson (BJ) damping.? Only the intermolecular part of the dispersion was used, the relevant parameters are given in the Supplementary material.

For non-symmetric complexes, i.e. in cases when the two interacting molecules in the complex are not equivalent, two choices of the fragment treated at a higher level of theory are possible in $\mathrm{QM} / \mathrm{MM}$ and embedding calculations. Since the two choices result in minor differences in the interaction energies, the arithmetic mean of the two calculations was used as result.

\subsection{Molecular systems}

The test systems of this study are illustrated on Fig. ??. In the discussion below, the distance of the monomers are measured as the distance between the centers of mass of the two fragments.

The pyrrole dimer was evaluated in six different relative orientations of the components. Three of them are co-planar (denoted as Pyr-Pyr (ILX)) with either identical alignment with the nitrogen atoms facing in the same direction $\left(\right.$ Pyr-Pyr (IL1)) or in a symmetrical $\mathrm{D}_{2 \mathrm{~h}}$ positioning with inward (Pyr-Pyr (IL2)) or outward (Pyr-Pyr (IL3)) pointing N-H groups. In addition, two T-shaped (Pyr-Pyr (T1)) and (Pyr-Pyr (T2)) and one stacked (Pyr-Pyr (S)) orientations were considered. In the latter, the fragments were aligned with dipole moments pointing predominantly opposite directions, but one of the molecules rotated in-plane by 10 degrees away from the $\mathrm{C}_{2 \mathrm{~h}}$ structure to lower the symmetry. The cytosine-uracil complex was also calculated in a stacked (sandwich) setup (Cyt-Ura $(S))$ with the oppositely oriented 
dipole moments and the line connecting the subsystems' centers of mass perpendicular to the molecular planes.

These seven cases cover a broad spectrum of the nature of intermolecular interactions in nitrogen-containing heterocycles with a large variety of equilibrium separations, making it possible to draw generic conclusions on the description of complexes of this type.

The equilibrium structures of the fragments, optimized at the MP2/6-31G* level, have been taken from Ref. ? . The structures used in the calculations are documented in Supplementary material. 

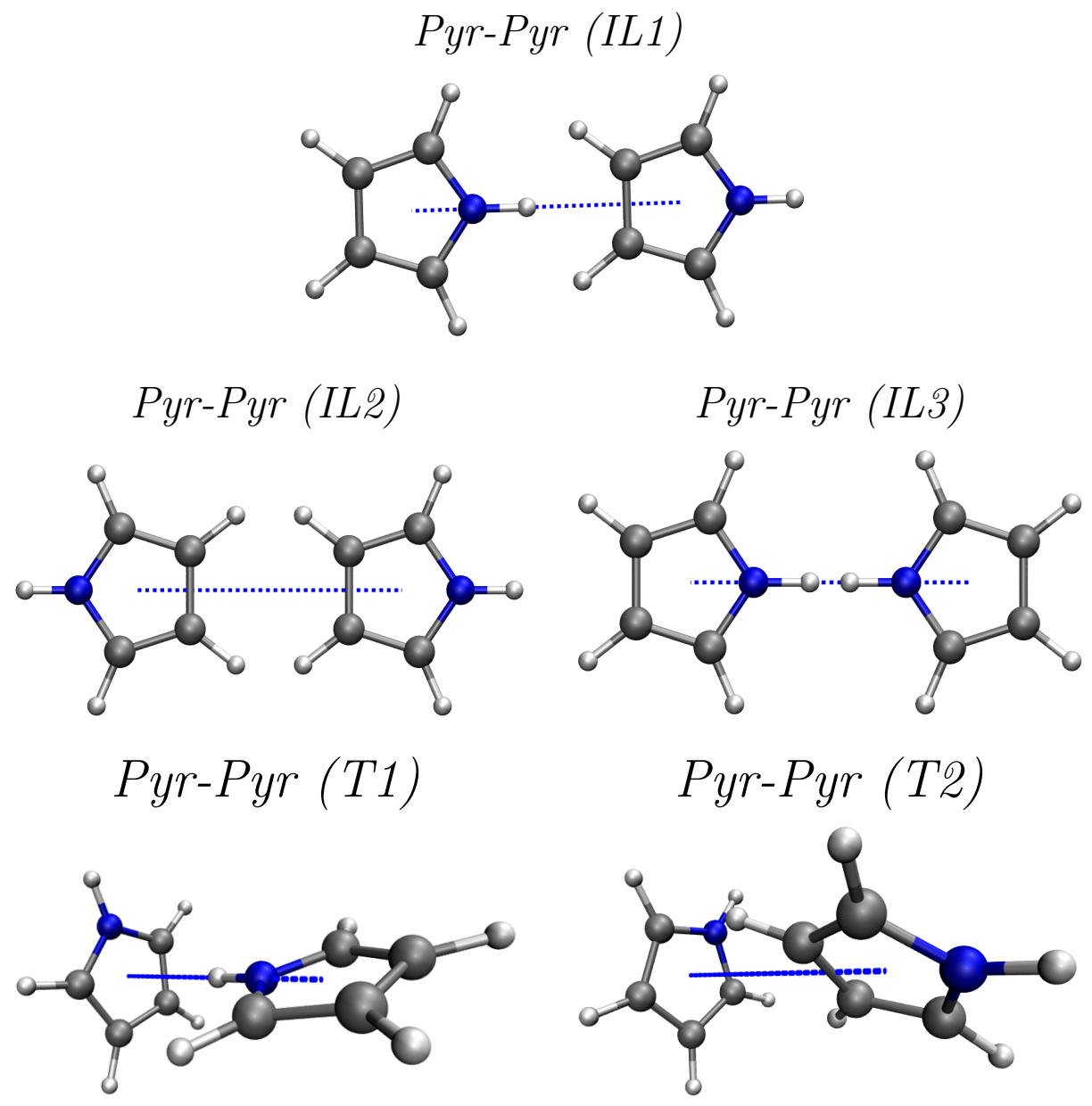

$$
\text { Pyr-Pyr (S) }
$$

Cyt-Ura (S)
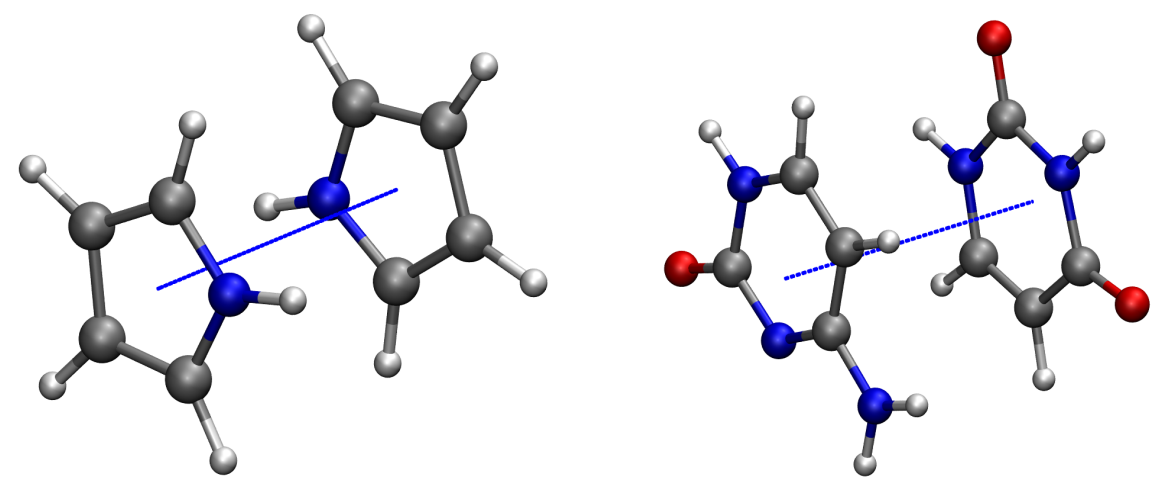

Figure 1: Orientation of the molecules in the test systems used in this study. The measure of the distance is represented by the blue dotted line connecting the centers of mass of the fragments. 


\section{Results and discussion}

\subsection{Dispersion and Pauli repulsion}
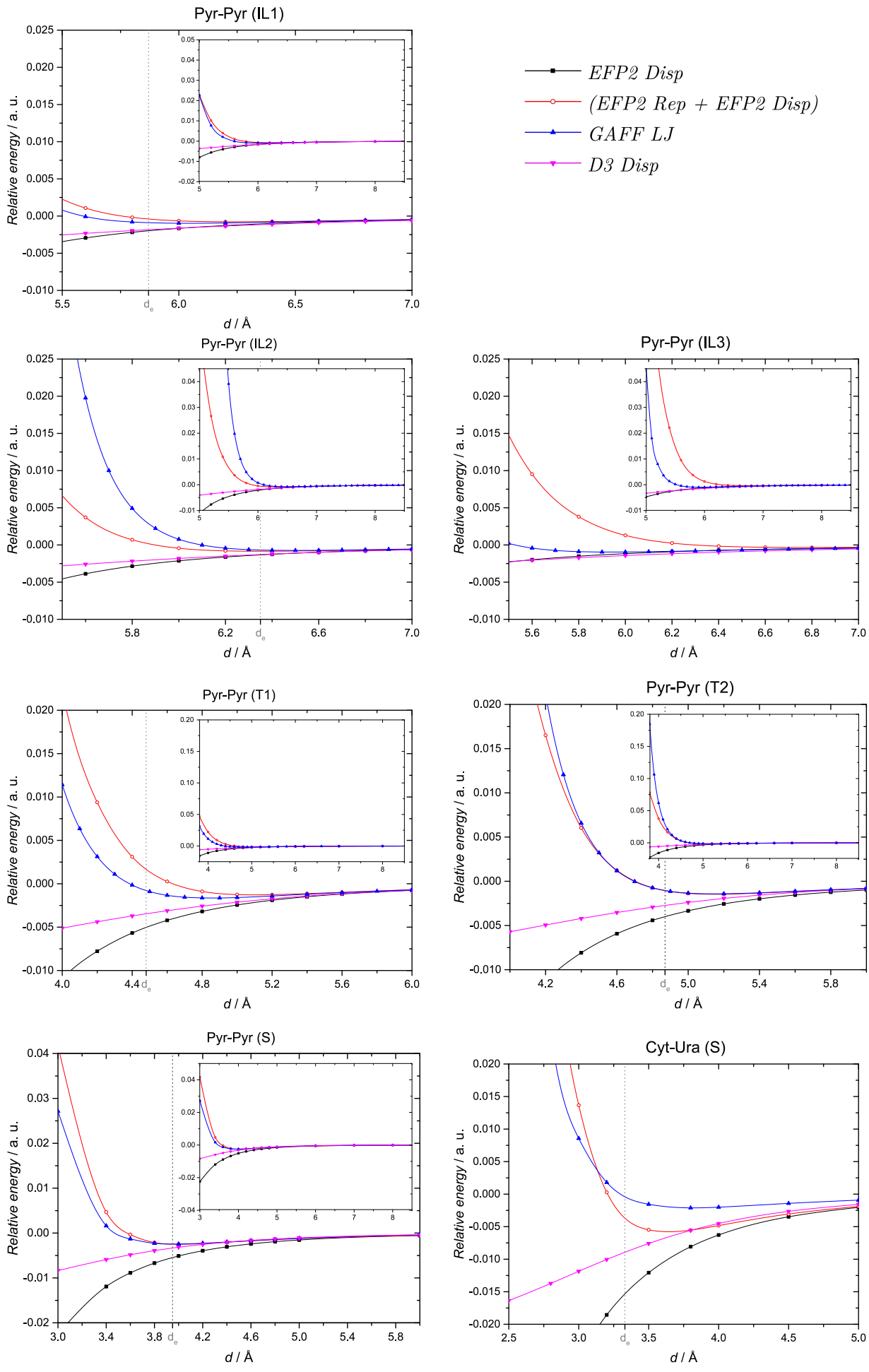

Figure 2: Distance dependence of various potentials describing dispersion and Pauli-repulsion contribution of the interaction energy. $d_{e}$ marks the reference equilibrium distance. 
In Figure ?? the dispersion contribution of the EFP2 method (EFP2 Disp, black) and D3 (D3 Disp, purple), as well as the EFP2's dispersion plus Pauli repulsion ((EFP2 Rep + EFP2 Disp), red) and Lennard-Jones potential of the GAFF force field (GAFF LJ, blue) are compared. As of the two dispersion curves, one can observe that the EFP2 ones are significantly steeper than the D3 curves. Consequently, for systems with a larger distance between the closest atoms of the different fragments these two choices EFP2 and D3 will give similar results, while for systems with a shorter separation the D3 dispersion contribution will be smaller, resulting in less attractive or more repulsive total interaction curves (see later). In our case this means that the choice between D3 and EFP2 is less relevant for the coplanar structures, while for the T-shaped and sandwich configurations this difference can be as large as 0.002 a.u. or even larger (0.01 a.u.) as in case of Cyt-Ura (S).

The dispersion and Pauli repulsion terms of EFP2 combined (EFP2 Rep + EFP2 Disp) correspond to, as also seen in Table ??, the effects covered by the GAFF Lennard-Jones potential. The behavior of the GAFF LJ contribution (blue curves on Fig. ??) with respect to the corresponding EFP2 curves (red curve) is not systematic. While EFP2 is generally more repulsive, for Pyr-Pyr (IL2) the opposite is true. Although the general shape of the curves appears to be similar, substantial energy differences can occur even around the equilibrium distances of some structures. For the Pyr-Pyr (IL1) and Pyr-Pyr (IL2), as well as for Pyr-Pyr $(S)$ the difference is very small at the corresponding equilibrium distance, becomes, however, substantial on the repulsive side of the curve. In contrast, for Pyr-Pyr (IL3) and Pyr-Pyr (T1) where the proton attached to the $\mathrm{N}$ atom is close to the other molecule, the discrepancy is remarkably large. Cyt-Ura $(S)$ is special in the sense that the two curves cross each other: at the equilibrium separation the $($ EFP2 Rep + EFP2 Disp) shows a relatively strong binding (0.005 a.u.) compared to the other examples in this study, while the GAFF LJ curve is less bound with an interaction energy closer to that of other structures (-0.002 a.u.). This means that for this system the dispersion contribution in GAFF LJ should be small, similarly to D3. This finding warrants the favorization of EFP2 potentials over Lennard-Jones type ones for the dispersion contribution in similar systems. One has to keep in mind, however, that other force fields can possibly show a better performance than GAFF LJ parameters. 


\section{$5.2 \quad$ Electrostatic interaction}
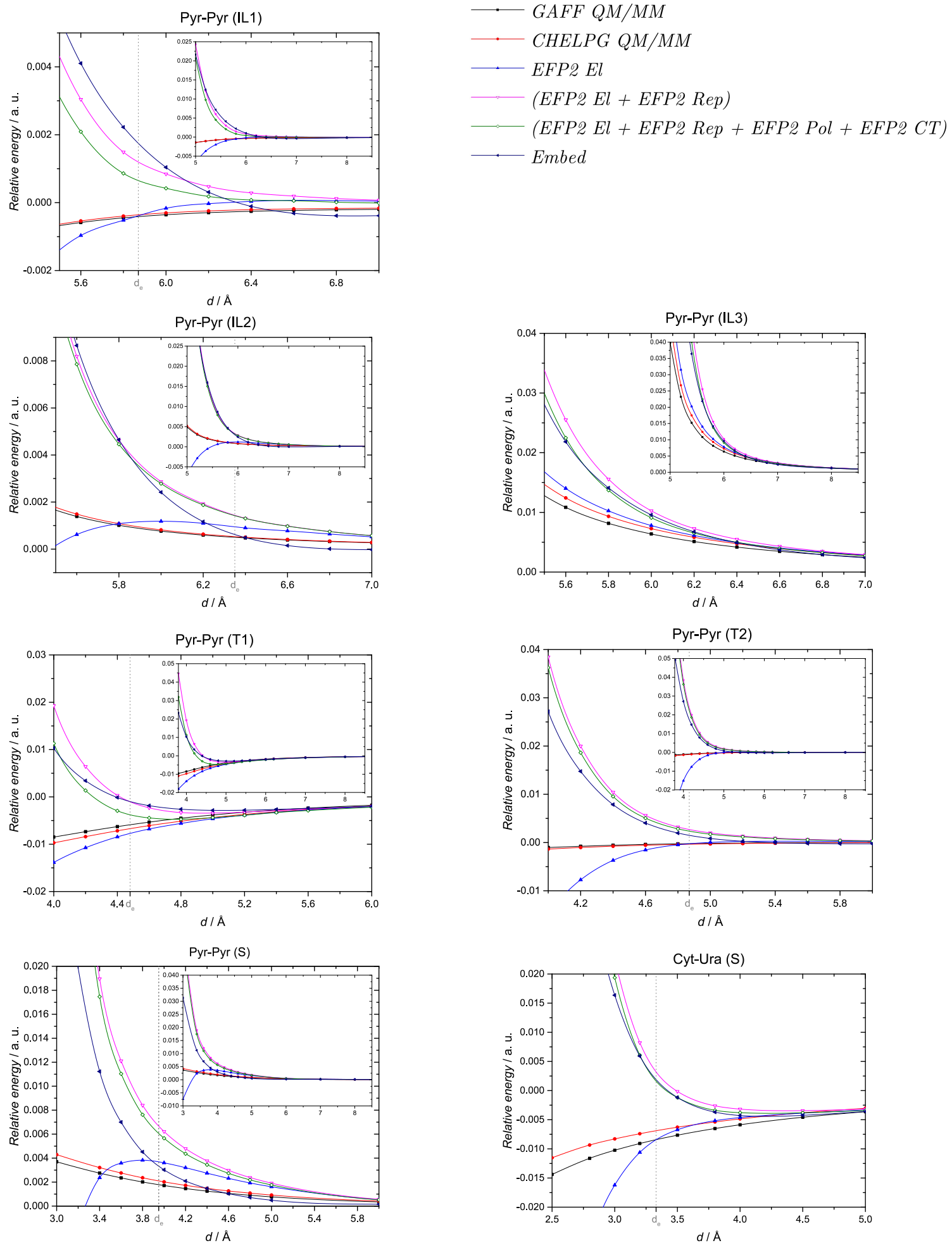

Figure 3: Distance dependence of various potentials describing electrostatic and Paulirepulsion contribution of the interaction energy. $d_{e}$ marks the reference equilibrium distance. 
In Figure ?? the distance dependence of the pure electrostatic contributions obtained from the QM/MM approach with GAFF Force Field (GAFF QM/MM, black), as well as CHELPG charges (CHELPG QM/MM, red) and the corresponding EFP2 part (EFP2 El, blue) are seen, along with the curve from the embedding calculation (which includes also Pauli repulsion, see above) (Embed, purple) and the EFP2 curve corresponding to it ((EFP2 El $+E F P 2$ Rep), magenta). The figure also includes the EFP2 curves augmented with the polarization and CT contributions ((EFP2 El + EFP2 Rep + EFP2 Pol + EFP2 CT), green).

These latter terms do not turn out to be important in any case. In fact, with the exception of Pyr-Pyr (IL1) where their inclusion reduces the repulsive nature of the surface to some extent, the difference they make is practically negligible.

When considering the pure electrostatic contributions (EFP2 El and QM/MM curves) one should discuss the attractive and repulsive cases separately. Attractive are Pyr-Pyr (IL1), Pyr-Pyr (T1) and Pyr-Pyr (T2) as well as Cyt-Ura (S) curves. In these cases the $\mathrm{QM} / \mathrm{MM}$ interaction curves are close to linear, while higher order dependence is seen for EFP2. This latter effect is most probably due to the damping/screening effect built into EFP2 but not in our QM/MM approach. Thus, even if QM/MM electrostatic attraction is stronger at longer separations (Pyr-Pyr (IL1), Pyr-Pyr (T2), Cyt-Ura (S)) EFP2 always predicts stronger interaction at shorter distances. In the two cases possessing repulsive electrostatic interactions (Pyr-Pyr (IL2) and Pyr-Pyr (S)), the EFP2 El curve shows a pronounced turn-back at shorter distances, also probably caused by the damping/screening formula employed in this model. At larger distances, before this turn-back occurs, the EFP2 $\mathrm{El}$ interaction is considerably stronger than that predicted by the QM/MM models. The largest relative discrepancy between the electrostatic curves is observed for the Pyr-Pyr (S), but note that the electrostatic interaction here is very small compared to other cases. Still, this discrepancy will influence even qualitatively the total interaction curve (see later).

There is no substantial difference between the curves obtained in the QM/MM approach, using either CHELPG and GAFF point charges. Therefore, we chose CHELPG in our further studies as the parameters can be obtained from an ab initio calculation for the molecules in question.

Since the embedding model (Embed, purple curve of Figure ??) automatically includes 
the Pauli repulsion contribution (see above), the electrostatic part of the embedding cannot be compared directly to the pure electrostatic curves. Instead, comparisons have to be made to the $(E F P 2 E l+E F P 2 R e p)$ curves. In this regard, a very good agreement is observed for Cyt-Ura (S), Pyr-Pyr (T1), Pyr-Pyr (T2), and Pyr-Pyr (IL3), while for the other cases (PyrPyr (IL1), Pyr-Pyr (IL2), Pyr-Pyr (S)) the embedding predicts more attractive curves at longer distances and more repulsive ones at shorter distances. (Note that two of these cases show repulsive electrostatics.) The fact that this latter discrepancy cannot be explained by the damping/penetration contribution, supports the idea that the embedding scheme used here incorporates this effect, as well. Near the equilibrium, substantial (relative) difference is observed for these arrangements.

For the two sandwich configurations, the comparison of the Embed (purple) curve to other electrostatic ones reveals the ultimate importance of Pauli repulsion, changing the electrostatic interaction qualitatively, even resulting in a minimum in case of Cyt-Ura $(S)$ complex. 


\subsection{Total interaction energy}
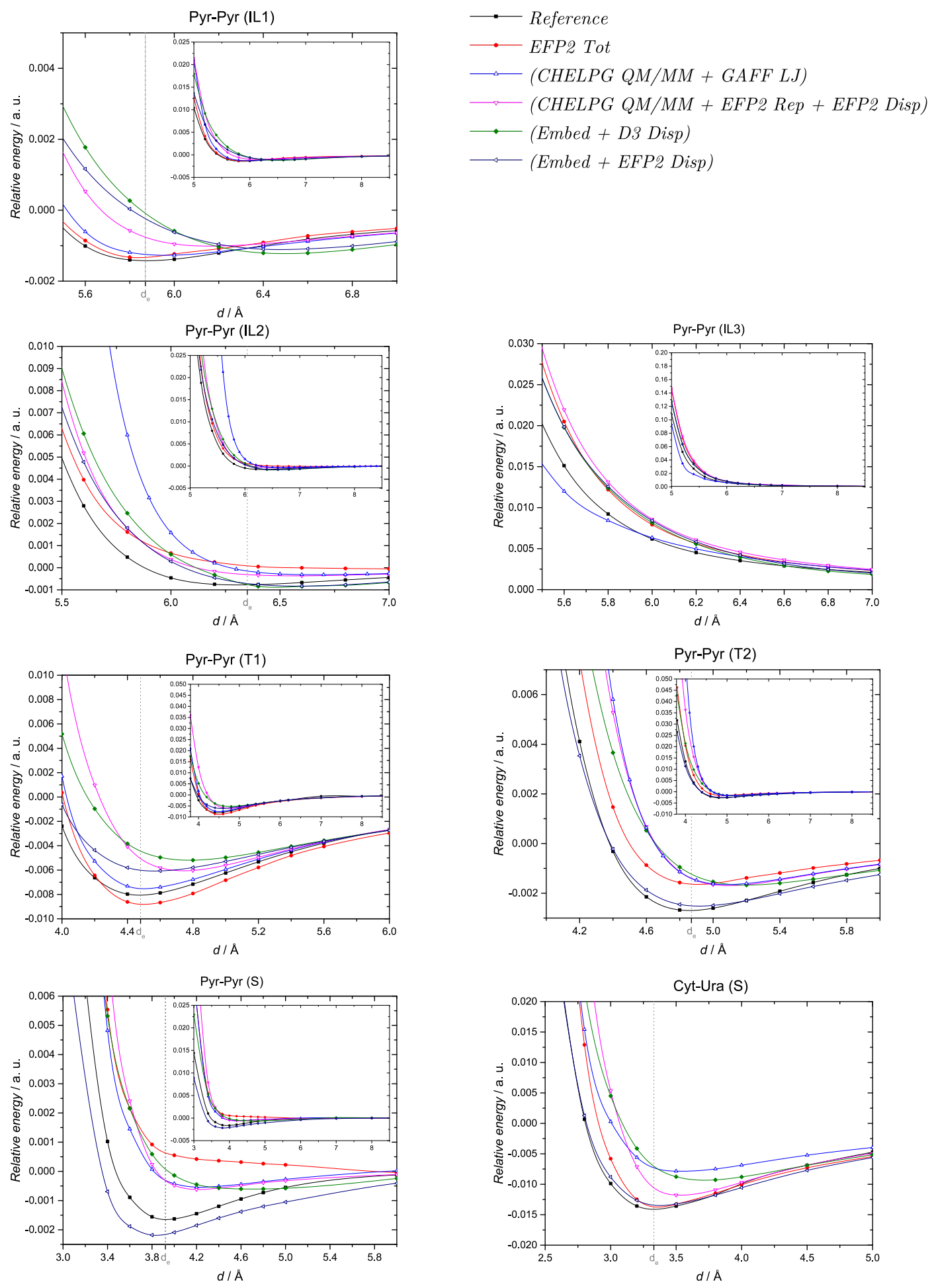

Figure 4: Total interaction energy of the investigated complexes calculated at various level of theory. $\mathrm{d}_{\mathrm{e}}$ marks the reference equilibrium distance. 


\begin{tabular}{|c|c|c|c|c|c|c|c|}
\hline & & & Pyr & Pyr & & & Cyt-Ura \\
\hline & $(I L 1)$ & (IL2) & (IL3) & $(T 1)$ & (T2) & $(S)$ & $(S)$ \\
\hline $\mathrm{d}_{e} /$ a.u. & 5.9 & 6.3 & $(6.0)^{a}$ & 4.5 & 4.9 & 3.9 & 3.3 \\
\hline Embed & 1.7 & 0.6 & 9.6 & -1.0 & 1.5 & 3.3 & 1.8 \\
\hline CHELPG $Q M / M M$ & -0.4 & 0.5 & 7.3 & -6.8 & -0.4 & 2.1 & -6.9 \\
\hline EFP2 $E l$ & -0.4 & 0.9 & 7.8 & -7.7 & -0.3 & 3.7 & -8.4 \\
\hline EFP2 Rep & 1.6 & 0.5 & 2.5 & 6.6 & 2.9 & 3.0 & 11.6 \\
\hline EFP2 Disp & -2.0 & -1.4 & -1.2 & -5.0 & -4.0 & -5.4 & -15.3 \\
\hline D3 Disp & -1.8 & -1.4 & -1.4 & -3.4 & -2.7 & -3.4 & -9.0 \\
\hline$G A F F L J$ & -0.9 & -0.7 & -0.9 & -0.8 & -1.1 & -2.4 & -0.4 \\
\hline$(E F P \mathscr{2} R e p+E F P 2 D i s p)$ & -0.4 & -0.9 & 1.3 & 1.6 & -1.1 & -2.4 & -3.7 \\
\hline EFP2 Tot & -1.3 & 0.1 & 7.9 & -8.8 & -1.6 & 0.6 & -13.7 \\
\hline$($ Embed + EFP2 Disp $)$ & -0.2 & -0.7 & 8.4 & -6.0 & -2.5 & -2.2 & -13.4 \\
\hline$($ Embed + Dз Disp $)$ & -0.1 & -0.8 & 8.2 & -4.5 & -1.2 & 0.1 & -7.0 \\
\hline Reference & -1.4 & -0.8 & 6.2 & -8.1 & -2.7 & -1.7 & -14.1 \\
\hline
\end{tabular}

a Selected point as there is no minimum in the investigated range.

Table 2: Energy contributions (in $\mathrm{mE}_{\mathrm{h}}$ ) of different terms and combinations at the reference equilibrium point $d_{\mathrm{e}}$.

In Figure ?? the total interaction energy of the investigated complexes are shown. The black curve is the reference energy calculated for the complex using the CCSD $(\mathrm{T})$ method and augcc-pVDZ basis with CP correction. The total EFP interaction energy EFP2 Tot is shown in red, the embedding curves augmented with two different dispersion corrections in green $($ Embed + D3 Disp $)$ and purple (Embed + EFP2 Disp), while the CHELPG QM/MM electrostatics, augmented with either the GAFF Lennard-Jones potential (CHELPG QM/MM + GAFF LJ) or the EFP2 dispersion and Pauli repulsion terms (CHELPG QM/MM+ EFP2 Rep + EFP2 Disp), are displayed in blue and magenta, respectively. The substantial differences in the shape of the presented curves warrant the individual discussion of the results for each test system. Additional information for this analysis is given in Table ?? where the different contributions to the total interaction energy are listed at the respective equilibrium distance of the complex under study.

Pyr-Pyr (IL1) structure: Despite the fact that the dipole moments of the two monomers 
are aligned in this system, there is a very weak interaction between the monomers. Contrary to e.g. the sandwich systems discussed below, here all three contributions to the interaction energy are small (see Table ??). Both the EFP2 Tot and the (CHELPG QM/MM+GAFF $L J)$ curves agree very well with the reference one, while the $(C H E L P G Q M / M M+E F P 2$ Rep + EFP2 Disp), as well as the dispersion corrected embedding curves, show a weaker agreement. The latter ones, in particular, show a minimum at a considerably longer distance, although the interaction energy at the minimum is similar to that of the reference. Contrary to the cases discussed below, here a replacement of the D3 dispersion correction with the EFP2 counterpart results in a negligible improvement. On the other hand, by pairing the CHELPG $Q M / M M$ term with EFP2 dispersion and Pauli repulsion instead of the GAFF $L J$, the agreement with the reference curve becomes worse and the result is closer to the embedding cases.

Pyr-Pyr (IL2) structure: The total interaction energy is even smaller in this structure than in Pyr-Pyr (IL1). This is partly due to the fact that the electrostatic part is repulsive, thus compensating dispersion. Here the best agreement with the reference is provided by $($ Embed + EFP2 Disp), although the minimum is at a somewhat larger distance. The (Embed + D3 Disp) combination provides a better location of the minimum point, but the difference is not relevant, and the short-distance repulsion is clearly overestimated by this model. The EFP2 Tot potential remains repulsive throughout the entire curve, thus failing to agree even qualitatively with the reference. The (CHELPGQM/MM+GAFF LJ) model is less bound than the reference, showing just the half of the interaction energy, however, the position of the minimum reasonably agrees with the $($ Embed + EFP2 Disp $)$ combination. Note that this is the system where a large discrepancy between the GAFF LJ and the (EFP2 Rep + EFP2 Disp) curves was observed above, the former being more repulsive.

Pyr-Pyr (IL3) structure: The total interaction curve for this system is repulsive in the investigated region, which is being reproduced by all methods. The best agreement with the reference is provided by the (CHELPG $Q M / M M+G A F F L J)$ combination. However, contrary to all other cases where the short-distance repulsion is clearly overestimated, this curve crosses the reference around $6 \AA$ separation and shows a considerably weaker repulsion below this point. This is in line with the finding that the GAFF LJ curve considerably 
underestimates the corresponding EFP2 curve (see Figure ??). The (Embed + EFP2 Disp) and (Embed + D3 Disp) combinations, as well as EFP2 Tot all agree very well, showing a similar error of parallelity to the reference: note that in this case the dispersion parts of the interactions were also found to agree above.

Pyr-Pyr (T1) structure: The total interaction energy in this system is the second largest among the investigated structures, with a strong attractive electrostatic component (see Table ??). (Note that since the two dipoles are perpendicular to each other, this part should be zero in the dipole approximation.) The absolute values of the Pauli repulsion and dispersion are also sizeable. The EFP2 Tot curve shows the best agreement with the reference, slightly overestimating the binding energy. The (CHELPG QM/MM+GAFF $L J)$ curve also comes close to the reference one and underestimates the binding by just $15 \%$, outperforming (CHELPG QM/MM +EFP2 Rep +EFP2 Disp). This deviation is clearly caused by the discrepancy of the GAFF LJ potential and the (EFP2 Rep + EFP2 Disp) terms discussed in section ??. With the combination of embedding and D3 (Embed + D3 Disp) the interaction energy is too weak by about 50\%, a large part of which can be corrected by replacing the D3 term with the EFP2 dispersion (Embed + EFP2 Disp).

Pyr-Pyr (T2) structure: The interaction in this structure is dominated by the dispersion, while the Pauli repulsion is mostly compensated by the electrostatic attraction. A good agreement is seen between most approximate methods, but they give only about half of the interaction energy. Embedding can again be improved by replacing D3 with EFP2 dispersion, resulting in an almost perfect agreement with the reference. Since GAFF LJ and (EFP2 $R e p+E F P 2$ Disp) were found to agree very well in this case (see section ??), the CHELPG $Q M / M M$ result cannot be improved considerably by changing one to the other.

Pyrrole-pyrrole sandwich (Pyr-Pyr $(S))$ : Describing the interaction in this system is a special challenge for theoretical methods. ${ }^{?}$ Near the equilibrium, dispersion is the strongest contribution to the interaction energy $\left(-5 \mathrm{mE}_{\mathrm{h}}\right)$, but it is largely cancelled by the Pauli repulsion and the (also repulsive) electrostatics, resulting in a very small total interaction energy. It is thus an example where good result can only be achieved with a method where a delicate balance between the different contributions is present. Indeed, as Figure ?? shows, there is a substantial discrepancy between the two embedding curves, with the one including 
D3 (Embed + D3 Disp) being less bonding while (Embed + EFP2 Disp) overbound. It is the latter which has the smaller absolute error (also the overall shape of the curve being similar to the reference), while the former is similar to both QM/MM curves. The agreement of the $\mathrm{QM} / \mathrm{MM}$ curves is a consequence of the similarity of the GAFF LJ and (EFP2 Rep + EFP2 Disp) potentials, discussed earlier in section ??. Still, these methods yield only about half of the reference total interaction energy. One should note, however, that this discrepancy is very small in absolute terms since this complex is loosely bound. Contrary to other cases, the EFP2 Tot curve is even qualitatively incorrect, showing a constantly repulsive intermolecular interaction. This unexpected behavior can be attributed to the overly strong electrostatic repulsion provided by the EFP2 method: note the very large discrepancy between the QM/MM and EFP electrostatics observed above. This system is thus an illustration of the extraordinary difficulty of modeling weak interaction in stacked $\pi-\pi$ complexes by approximate methods. In this regard, the good performance of the combination of embedding and EFP2 dispersion is remarkable.

Cytosine-uracil complex (Cyt-Ura $(S))$ : Among the studied systems, this complex shows the strongest interaction between the two molecules $\left(-14 \mathrm{mE}_{\mathrm{h}}\right)$. Similarly to Pyr-Pyr $(S)$, the interaction is dominated by the dispersion $\left(-12 \mathrm{mE}_{\mathrm{h}}\right)$ which is about twice as large as the size of the electrostatic and Pauli repulsion. Contrary to Pyr-Pyr $(S)$ however, the electrostatic part is attractive, compensating the Pauli repulsion almost entirely. In comparison to the reference curve, the EFP2 Tot as well as (Embed + EFP2 Disp) potentials turn out to be the best choices. At longer distances also the (CHELPG QM/MM+EFP2 Rep + EFP2 Disp) curve comes close to the reference, but the repulsion shown at the short-distance side of the minimum is clearly too strong. It is thus seen that for this system the ab initio methods using EFP2 components clearly outperform those with D3 or LJ corrections.

In summary, despite the very different bonding situations in the model systems, most of the methods investigated here give reasonable result.

The EFP2 Tot interaction curves agree very well with the reference curve in most cases, however this model fails even qualitatively for the Pyr-Pyr (S) and Pyr-Pyr (IL2) systems which are loosely bound by other methods but not with EFP2 Tot.

CHELPG QM/MM, with either the GAFF LJ or the (EFP2 Rep + EFP2 Disp) term 
gives similar results for the stacked systems and Pyr-Pyr (T2), while in other cases the curves with GAFF LJ correction are substantially closer to the reference curves, except for PyrPyr (IL2) where GAFF LJ fails badly. Typically, the bonding provided by this combination is a bit weaker than in the reference, representing a quality comparable to EFP2 Tot and somewhat less accurate than embedding methods.

The dispersion corrected embedding curves $($ Embed + EFP2 Disp) and $($ Embed + D3 Disp) are in most cases closer to the reference than other methods investigated here. From these two, the former with the EFP2 dispersion clearly gives the best agreement, outperforming the latter which uses D3 dispersion.

\section{Conclusions}

The aim of this study was to investigate the impact of various terms of approximate methods on the intermolecular interaction energy of the test systems and the performance of various combinations thereof. Despite the approximate, in some sense arbitrary nature of these contributions, we could confirm their importance in the description. In fact, it was found that all contributions of a particular model need to be included even for qualitatively correct potential energy curves.

With the goals of our future studies in mind, we pay special attention to the models that can be combined with arbitrary electronic structure methods. In this respect, QM/MM type formulations with point charges are ideal since their effect can easily be implemented in the one-electron Hamiltonian, without affecting the execution of any level of calculation. However, despite the widespread successful applications to e.g. solvent effects, it is not suited per se for loosely bonded dimers investigated here since other terms, such as dispersion or Pauli repulsion are not negligible, often even dominating the interaction. To cover these effects, the electrostatic part needs to be complemented with additive terms to the interaction energy. Most force fields include a Lennard-Jones type potential for his purpose, however, our calculations show that more sophisticated methods, e.g. the Effective Fragment Potential technique, can be better choices.

It needs to be understood though, that the various theories do not address the division of 
the interaction energy into these terms with the same strategy, therefore it is not a surprise that combinations of different approaches also tend to give inconsistent or controversial results. Nevertheless, certain setups show a promising performance, approaching the reference potentials to a reasonable extent.

In particular, the use of DFT/CCSD embedding to cover electrostatic and Pauli repulsion effects, with accounting for dispersion via the respective EFP2 term acts as a well working strategy in most cases, providing a remarkable accuracy for the stacked interactions. The QM/MM electrostatics using CHELPG charges, with the point charges placed on the partner molecule's nuclei is also a viable alternative to the formulation of widely adopted force fields, also liberating the description from the dependence on the system and the force field design. Combination with either the EFP2 dispersion and Pauli repulsion terms or a Lennard-Jones potential of the GAFF force field gave similar results, with the latter showing a slightly better performance in some examples. Nevertheless, this finding is likely to be specific to the choice of this particular FF and may not be achievable for systems with different atom types. Also considering the rather rhapsodic behavior of the LJ contribution, producing both severe under- and overestimation of the interaction energy for specific arrangements, its application is difficult to support. Overall, the authors of this paper feel that the idea of a general, system-independent description provided by EFP2 is comforting enough to be favored over the LJ approach of any force field.

The D3 correction, a strategy also founded on the concept of transferable atom type parameters, was clearly outperformed by the EFP2 dispersion term in our results. This finding is, however, not related to its applicability in DFT, its original purpose of design.

Among the many alternative formulations arising from the synthesis of different theories we thus find combinations which not only offer good performance, but also retain the generality and theoretical desirability of $a b$ initio modeling. The sophisticated description offered by DFT/CCSD embedding, with the missing terms sourced from EFP is a powerful setup in this set and most likely the recommended idea to be pursued in future studies on excited states. 


\section{Acknowledgements}

This work has been supported by the National Research, Innovation and Development Fund (NKFIA) Grant No. 124293. We thank Prof. Mihály Kállay and his coworkers for assisting the embedding calculations and making adjustments in the MRCC code. We also thank Prof. György Ferenczy for his helpful guidance about non-covalent interactions.

\section{Conflict of interest}

The authors declare no conflict of interest.

\section{Supporting Information}

Structures of the the investigated monomers and complexes, calculated CHELPG charges, employed parameters of the GAFF Force Field as well as $\mathrm{C}_{6}$ coefficients and damping parameters of the D3 correction are listed here. 